\title{
A scale for the psychological magnitude of number*
}

\author{
BRUCE SCHNEIDER, SCOTT PARKER, DAN OSTROSKY, DAVID STEIN, and GARY KANOW \\ Columbia University, New York, New York 10027
}

\begin{abstract}
A scale of the "psychological magnitude" of number was constructed from similarity ratings of the 45 number pairs that can be obtained from a set of 10 integers. A nonmetric analysis of these similarity ratings showed that "psychological number" was a power function of number.
\end{abstract}

In a magnitude-estimation task, Ss are asked to assign numbers to stimuli such that the numbers assigned are directly proportional to the psychological magnitudes of the stimuli. Stevens (1962) has shown that for a number of sensory continua, the numerical assignments obtained in this fashion are a power function of stimulus intensity, i.e.,

$$
\mathrm{N}=\mathrm{kI}^{\mathrm{n}} \text {, }
$$

where $\mathrm{N}$ represents $\mathrm{S}$ 's numerical judgment, I denotes the physical value of the stimulus, $n$ is the exponent of the power function, and $\mathrm{k}$ is a constant of proportionality. If it is assumed that Ss are actually assigning numbers that are proportional to psychological magnitude $(\mathrm{N}=\mathrm{a} \Psi$, where $\Psi$ represents psychological magnitude or sensation), then it can be argued that psychological magnitude is a power function of stimulus intensity, and that the value of the exponent of the power function is obtained directly from the magnitude estimation data. Several investigators, however, have recently become concerned with this assumption of proportionality, and have argued that Ss use numbers in a nonlinear fashion when judging sensory events (Attneave, 1962; Curtis et al, 1968; Ekman \& Hosman, 1965; Rule, 1969, 1971, 1972; Rule \& Curtis, 1973; Rule et al, 1970). The present experiments are an attempt to determine how people perceive and use numbers to measure or scale subjective events and if, indeed, they use them in a nonlinear fashion. This, in effect, requires the development of a scale of psychological number.

Developing a numerical representation of psychological number requires a scaling technique which is capable of scaling numbers. Such a technique must avoid the S's knowledge of the properties of numbers and should make minimal assumptions about the nature of the S's judgments. Recent work by Shepard (1966) on nonmetric scaling supplies the basis for such experiments.

The Ss were requested to rate the similarity of pairs of

\footnotetext{
*We would like to thank Norma Graham and Donald Hood for their helpful comments and suggestions. Pilot experiments were performed at Marymount Manhattan College. This research was supported by NSF Grant G B 36211.
}

numbers on a scale of 1 to 9 . The use of similarity judgments as a scaling technique for numbers prevents certain obvious strategies on the part of the $S$, based on his knowledge of numbers. For instance, if the Ss were asked how different or unlike two numbers were, they would most likely respond by giving the subtractive difference between two numbers. Even if they were told to rate how different the numbers were on a scale of 1 to 9, they could still (1) compute the subtractive difference, and (2) develop a one-to-one correspondence between certain ranges of magnitude of subtractive difference and the numbers on the rating scale. Consequently, any scale of psychological number constructed from such data would reflect the properties of the number system, i.e., psychological number would be proportional to number. However, when similarity judgments are involved, there is no obvious and easily computed property of numbers that could be used to determine their similarity. Hence, judgments of the similarity of numbers should reflect the psychological proximity of one number to another on a psychological scale. And, as Shepard (1966) has shown, all that is needed to construct an interval scale representation of stimuli is the rank order of the proximity measures taken on all possible pairs of stimuli in question. These proximity measures are assumed to be a monotonic decreasing function of the distances between the stimuli along the psychological continuum, i.e., as distance increases, proximity decreases. Since the rank order of interpoint distances along a line segment is sufficient to determine a numerical representation of the stimuli which is unique up to a línear transformation, an interval scale of psychological number can be constructed from judgments of the similarity of pairs of numbers.

\section{METHOD}

\section{Subjects}

Twenty undergraduates associated with Columbia University served as Ss in this experiment.

\section{Procedure}

Two conditions were employed. In Condition 1, similarity judgments were obtained for the 45 possible number pairs that can be formed from the following 10 numbers: $17,59,92,121$, $148,174,215,263,270,306$. Note that the arithmetic spacing between numbers is fairly uniform. A second set of 10 numbers, 
where the logarithmic spacing is approximately uniform $(17,24$, $33,46,61,90,129,177,245,312$ ), was used to generate the 45 number of pairs in Condition 2. Ten Ss were employed in each condition. Each $S$ served in three experimental sessions. In the first session, the Ss were presented, once in irregular order, with the $\mathbf{4 5}$ distinct pairs of numbers which could be formed from the set of 10 . In the second and third sessions, the 45 number pairs were presented twice, in irregular order, with a 10 -min break separating the presentations. The Ss in both conditions rated similarity of number pairs on a 9-point scale. At the beginning of the first session, the following set of instructions was read to the $S$. "This is an experiment to determine how similar numbers seem to you. When the experiment begins, you will be presented with pairs of numbers. These number pairs will be read to you. Your task will be to evaluate how similar the numbers in a pair are by assigning their similarity a category from 1 to 9 . The categories represent degrees of similarity. Category 9 is to be used for the highest degree of similarity, while Category 1 should be used for the lowest degree of similarity. For example, 79 and 84 belong in Category 9 because they are highly similar. Category 1 , on the other hand, is reserved for pairs which have low similarity, such as 14 and 337. When you hear a pair of numbers, assign it to one of the nine categories so that the greater the similarity, the higher the category to which it is assigned. You should feel free to use any category as many times as you wish." These instructions were repeated at the beginning of the second and third sessions, if requested by the $S$.

\section{RESULTS}

The basic data in this experiment consisted of five similarity judgments of each pair of numbers by each $\mathrm{S}$. Estimates from the first session were discarded. The arithmetic mean of the remaining four estimates of similarity by an $S$ was computed for each of the 45 stimulus pairs. The arithmetic means were then ranked, within Ss, from 1 to 45 . Kendall's coefficient of concordance $(\mathrm{W})$, which measures the degree of ordinal agreement in the ratings, was found to be 0.91 in Condition 1 and 0.92 in Condition 2. A W value of 1.0 indicates perfect ordinal agreement. Thus, agreement among Ss as to the rank order of number similarity was good.

The arithmetic mean of the ranks across Ss was computed for each stimulus pair. These mean ranks were reversed to obtain an ordinal index of the psychological distance between the numbers and used as an input to a nonmetric scaling computer program (Carvellas \& Schneider, 1972). The output from the program is a set of 10 numbers which represent projection values for the stimuli along a line segment. The projection values are unique up to multiplication and addition of a constant, i.e., represent interval scale measurement.

Stress, Kruskal's (1964) measure of goodness of fit, was computed for the projection values. Stress measures the discordance between the predicted distances, ds, and a set of distances, $\hat{\mathrm{d}}$, that are (1) monotonically related to the original distances (i.e., preserve the rank ordering), and (2) as much like the ds as they can be within the restrictions imposed by (1). Stress is given by $\left[\Sigma(\mathrm{d}-\hat{\mathrm{d}})^{2} / \Sigma \mathrm{d}^{2}\right]^{1 / 2}$ often expressed as a percentage. Notice that perfect ordinal agreement produces $d=\hat{d}$, and, in this case, stress $=0$. The stress values were $2.1 \%$ for Condition 1 (linear spacing) and $2.4 \%$ for Condition 2 (log spacing). Kruskal states that stress values of $2.5 \%$ or less indicate excellent agreement between ds and ds. Young (1970) has shown that with 10 stimuli, and with stress values as small as these, the projection values can be regarded as an interval scale representation of the true values of the stimuli.

Attneave (1962) suggested that psychological number might be a power function of number. To check Attneave's conjecture, it is necessary to adjust the projection values by adding a constant. Since the projection values, $\mathrm{P}_{\mathrm{i}}$, obtained from the nonmetric program provide an interval scale of psychological number, they are unique only up to addition and multiplication by a constant. Therefore, the true values of "psychological number" should be linearly related to the projection values, i.e., $\Psi_{i}=a_{a}+b$, where $\Psi_{i}$ is the psychological magnitude of the number $N_{i}, P_{i}$ is the projection value for the number $\mathrm{N}_{\mathrm{i}}$, and $\mathrm{a}$ and $\mathrm{b}$ are constants. If a power function relates psychological number to number, then $\Psi_{i}=\mathrm{kN}_{\mathrm{i}}{ }^{\mathrm{n}}=\mathrm{aP}_{\mathrm{i}}+\mathrm{b}$. Equivalently, $P_{i}+(b / a)=(k / a) N_{i}{ }^{n}$, or $P_{i}+b^{\prime}=k^{\prime} N_{i}{ }^{n}$, where $b^{\prime}$ is unknown. Taking logarithms on both sides of the equation, a power function describes the present data if there is a $b^{\prime}$ such that $\log \left(\mathrm{P}_{\mathrm{i}}+\mathrm{b}^{\prime}\right)=n \log \left(\mathrm{N}_{\mathrm{i}}\right)+$ $\log \left(\mathrm{k}^{\prime}\right)$, i.e., if for some $\mathrm{b}^{\prime}, \log \left(\mathrm{P}_{\mathbf{i}}+\mathrm{b}^{\prime}\right)$ is linear with $\log$ number. A value of $b^{\prime}$ was therefore sought that would maximize the squared correlation coefficient $\left(\mathrm{r}^{2}\right)$ between $\log \left(\mathrm{P}_{\mathrm{i}}+\mathrm{b}^{\prime}\right)$ and $\log \left(\mathrm{N}_{\mathrm{i}}\right)$. The projection values, adjusted by the values of $\mathbf{b}^{\prime}$ which maximized $\mathrm{r}^{2}$ and rescaled (multiplied by a constant) so that a projection value of 1 corresponded to the number 1 , are called the adjusted number projections.

Figure 1 (Panels $a$ and $b$ ) shows plots of adjusted number projections as a function of number (log-log coordinates) for both Conditions 1 and 2. Best fitting straight lines, determined by the method of least squares, are drawn in each panel. In both cases, a linear relationship provides a good fit to the data $\left(\mathrm{r}^{2}=0.998\right.$ and 0.994 for Conditions 1 and 2, respectively). However, the slope of the line (exponent of the power function) differs slightly for the two cases $(0.69$ for linear spacing and 0.80 for log spacing). In other words, the spacing of the stimuli does seem to have an effect on the value of the exponent.

To check whether a logarithmic relationship between the adjusted number projections and number provided a better fit to the data than a power function, the adjusted number projections were plotted as a function of number in semilog coordinates for both Conditions 1 and 2 (Fig. 1, Panels $c$ and d). A straight line in these coordinates would indicate that psychological number was a logarithmic function of number (Fechner's law; cf. Stevens, 1962). Best fitting straight lines, determined by the method of least squares, are drawn in these panels. It is clear that the adjusted number projections in Panels $\mathrm{c}$ and $d$ do not fall on the best fitting line, but rather are a 
Fig. 1. Adjusted number projections (see text) as a function of number for two different sets of stimuli. Notice that the ordinate is spaced logarithmically in Paneis a and $b$, and arithmetically in Panels $c$ and $d$.

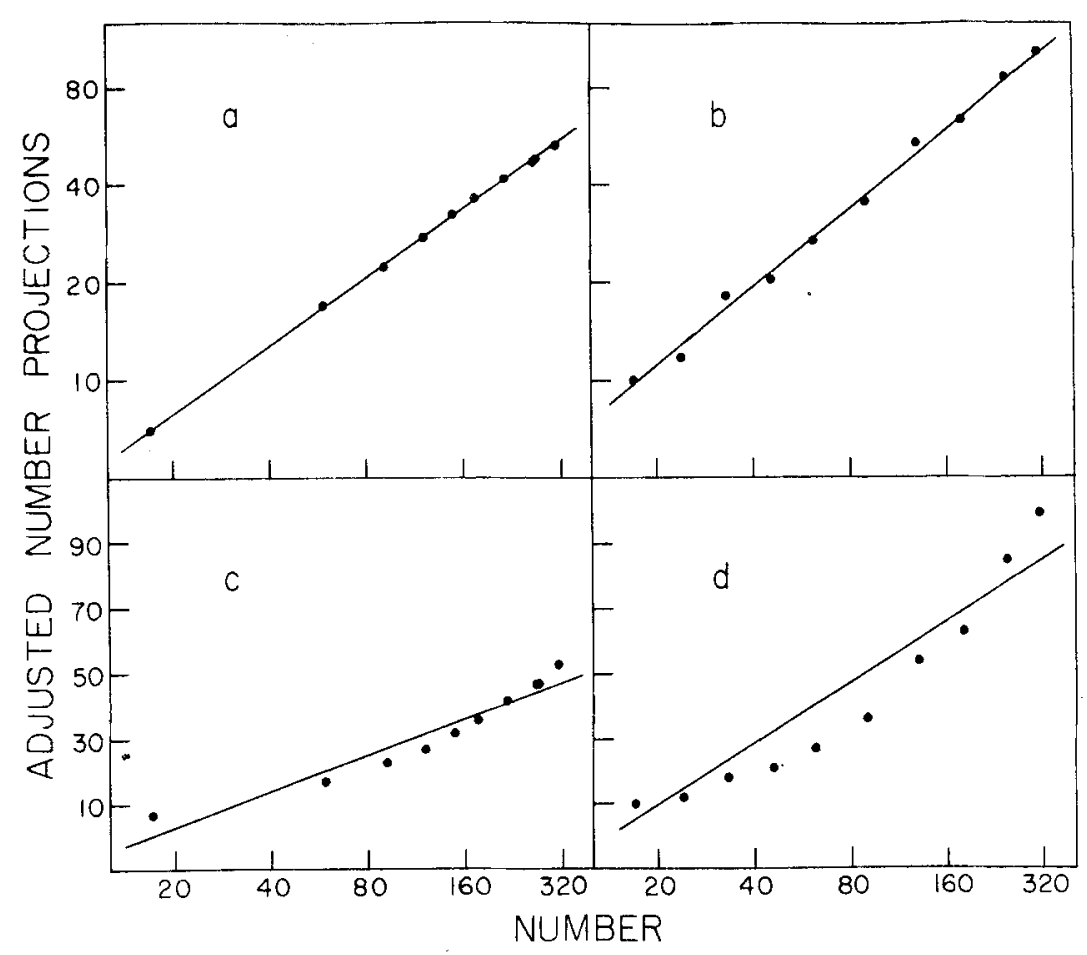

positively accelerated function of $\log$ intensity. Values of $\mathrm{r}^{2}$ for Panels $\mathrm{c}$ and $\mathrm{d}$ are 0.916 and 0.917. Figure 1, then, shows that a power function provides a better description of the relationship between the adjusted number projections and number than does a logarithmic function.

\section{DISCUSSION}

To the extent that an S's judgment of the similarity of two numbers is monotonic with the "psychological proximity" of one number to another, the present results indicate that psychological number is a power function of number. Informal interrogation of the Ss after the experiment did seem to support this assumption. First, none of the Ss indicated that they were using any numerical strategies, i.e., they were not dividing, subtracting, etc. Second, their latencies to a number pair were, in general, quite short. Hence, it is not unreasonable to hold that the similarity judgments were monotonically related to the psychological proximity of one number to another.

If, as this study indicates, psychological number is a power function of number (with an exponent in the range 0.7-0.8), it implies that in experiments where Ss are required to estimate directly the psychological magnitude of stimuli, the value for the exponent of that continuum is conssistently overestimated. Consider for the moment a model in which magnitude estimation is a form of cross-modality matching (Attneave, 1962) where the S matches "psychological number" to the magnitude of the sensation. If both psychological number and sensation magnitude are power functions of number and stimulus intensity, respectively, a match between the two $\left(\Psi_{\mathrm{N}}=\Psi_{\mathrm{s}}\right.$ implies $\mathrm{N}^{0.75}=\mathrm{I}^{\mathrm{m}}$, where $\Psi_{N}$ is the psychological number, $\Psi_{s}$ is the sensation magnitude associated with the intensity (I) of the stimulus. The expression $\mathrm{N}^{\mathbf{0 . 7 5}}=\mathrm{I}^{\mathrm{m}}$ is equivalent to $\mathrm{N}=$ $\mathrm{I}^{\mathrm{m} / 0.75}$. This implies that the exponent obtained in a magnitude estimation experiment is actually equal to the true exponent $(\mathrm{m})$ divided by the exponent for the number continuum (0.75). Hence, in order to obtain the true exponent for a continuum, one should multiply the obtained exponent by 0.75 . A weak prediction of this formulation is that the true value of the exponent for a sensory continuum should be smaller than that indicated by magnitude estimation experiments. Sensory scales constructed from nonmetric analyses of difference and similarity estimates (Parker \& Schneider, 1974; Rule et al, 1970) as well as nonmetric analyses of combined stimulus effects (LeVelt et al, 1972; Rule \& Curtis, 1973) also arrive at a power function representation. However, the exponents of the power functions are consistently lower than those obtained from straightforward magnitude estimation experiments, a result that is consistent with the formulation presented here.

It should be noted that this model of magnitude estimation was first advanced by Attneave (1962) and pursued by Curtis and Rule in a number of studies (cited above). According to the Attneave model, magnitude estimation consists of two stages. In the first stage, stimuli are mapped into psychological magnitudes or sensations. In the second stage, the psychological 
magnitudes are mapped into the number continuum. Attneave assumed that both transformations, stimuli to sensations and sensations to numbers, were power functions. The present results offer support for this two-stage theory of magnitude estimation.

However, while the present results are consistent with the two-stage theory, they cannot account for the individual differences which are often observed in direct scaling experiments. It is quite possible that the differences in exponents obtained in magnitude estimation experiments for different $S s$ are due to different psychological number scales rather than differences in the underlying representation of signals. If this is indeed so, then number scales developed for individual Ss should show a relatively wide range in thevalue of the exponents, a range large enough to account for the observed variation in exponents from person to person in magnitude-estimation experiments.

\section{REFERENCES}

Attneave, F. Perception and related areas. In S. Koch (Ed.), Psychology: A study of a science. Vol. 4. New York: McGraw-Hill, 1962.

Carvellas, T., \& Schneider, B. Direct estimation of multidimensional tonal dissimilarity. Journal of the Acoustical Society of America, 1972, 51, 1839-1848.
Curtis, D. W., Attneave, F., \& Harrington, T. L. A test of a two-stage model of magnitude judgment. Perception \& Psychophysics, 1968, 3, 25-31.

Ekman, G., \& Hosman, B. Note on subjective scales of number. Perceptual \& Motor Skills, 1965, 21, 101-102.

Kruskal, J. B. Multidimensional scaling by optimizing goodness of fit to a nonmetric hypothesis. Psychometrika, 1964, 29 1-27.

Levelt, J. M., Riemersma, J. B., \& Burt, A. A. Binaural additivity of loudness. British Journal of Mathematical \& Statistical Psychology, 1972, 25, 51-68.

Parker, S., \& Schneider, B. Nonmetric scaling of loudness and pitch using similarity and difference estimates. Perception \& Psychophysics, 1974, 15, 238-242.

Rule, $S$. J. Equal discriminability scale of number. Journal of Experimental Psychology, 1969, 79, 35-38.

Rule, S. J. Discriminability scales of number for multiple and fractional estimates. Acta Psychologica, 1971, 35, 328-333.

Rule, S. J. Comparisons of intervals between subjective numbers. Perception \& Psychophy sics, 1972, 11, 97-98.

Rule, S. J., Curtis, D. W., \& Markley, R. P. Input and output transformations from magnitude estimation. Journal of Experimental Psychology, 1970, 86, 343-349.

Rule, S. J., \& Curtis, D. W. Conjoint scaling of subjective number and weight. Journal of Experimental Psychology, 1973, 97, 305-309.

Shepard, $\dot{R}$. N. Metric structures in ordinal data. Journal of Mathematical Psychology, 1966, 3, 287-315.

Stevens, S. S. The surprising simplicity of sensory metrics. American Psy chologist, 1962, 17, 29-39.

Young, F. W. Nonmetric multidimensional scaling: Recovery of $\mathrm{m}$ etric inform ation. Psychometrika, 1970, 35, 455-473.

(R eceived for publication January 11, 1974; revision received February 20, 1974.) 